\title{
Full characterization of the hydrodynamic boundary condition at the atomic scale using an oscillating channel: Identification of the viscoelastic interfacial friction and the hydrodynamic boundary position
}

\author{
Takeshi Omori $\odot^{*}$ and Naoki Inoue \\ Department of Mechanical Engineering, Osaka University, 2-1 Yamadaoka, Suita, Osaka 565-0871, Japan \\ Laurent Joly $₫$ and Samy Merabia $\odot$ \\ Université de Lyon, Université Claude Bernard Lyon 1, CNRS, Institut Lumière Matière, \\ F-69622 Villeurbanne, France \\ Yasutaka Yamaguchi $\odot$ \\ Department of Mechanical Engineering, Osaka University, 2-1 Yamadaoka, Suita, Osaka 565-0871, Japan \\ and Water Frontier Science \& Technology Research Center (W-FST), Research Institute for Science \\ \& Technology, Tokyo University of Science, 1-3 Kagurazaka, Shinjuku-ku, Tokyo 162-8601, Japan
}

(Received 28 May 2019; published 1 November 2019)

\begin{abstract}
Flows in nanofluidic systems are controlled by the hydrodynamic boundary condition (BC), involving the friction coefficient and the hydrodynamic wall position. Here we considered a liquid nanoslab confined between two walls, where we derived, from the Stokes equation and the Navier slip BC, analytical expressions for the liquid response to an oscillatory tangential motion of the walls in terms of the wall shear stress and mean fluid velocity. By fitting these expressions to molecular dynamics simulation results, we could extract both the viscoelastic friction coefficient and hydrodynamic wall position for walls with three different wettabilities, hence fully characterizing the frequency-dependent hydrodynamic boundary condition. The proposed method could be applied to a variety of liquid-solid interfaces of interest, e.g., for flows of complex fluids or fluids at a low temperature. It should also support methodological developments on the characterization of the hydrodynamic slip in general.
\end{abstract}

DOI: 10.1103/PhysRevFluids.4.114201

\section{INTRODUCTION}

In order to solve the continuum equations of motion for a fluid in the presence of walls, one has to specify a hydrodynamic boundary condition (BC) on the wall. Based on the continuity of stress at the fluid-solid interface, Navier [1] proposed that the viscous shear stress in the fluid at the wall surface, $\eta \partial u / \partial z$ (with $\eta$ the shear viscosity, $z$ the normal to the interface, and $u$ the velocity parallel to the interface), is equal to an interfacial friction per unit wall area $\tau$. He assumed a fluidlike friction, where $\tau$ is proportional to the slip velocity $u_{s}$ (i.e., the jump of parallel velocity across

\footnotetext{
*t.omori@mech.eng.osaka-u.ac.jp

Published by the American Physical Society under the terms of the Creative Commons Attribution 4.0 International license. Further distribution of this work must maintain attribution to the author(s) and the published article's title, journal citation, and DOI.
} 
the interface): $\tau=\lambda u_{s}$, defining the fluid-solid friction coefficient (FC) $\lambda$. The Navier BC involves two free parameters, the FC $\lambda$ and the hydrodynamic position of the wall surface, $z_{s}$, where the BC applies [2], i.e., where the slip velocity and wall shear rate are defined. This position differs in general from the physical position of the wall surface [3-7]. The Navier BC is often rewritten as a kinematic relation on the velocity field, and referred to as the partial slip BC: $u_{s}=\left.b(\partial u / \partial z)\right|_{z=z_{s}}$, where $b=\eta / \lambda$ is the so-called slip length.

Experimental tests of the hydrodynamic BC at the time of Navier could not detect any slip, and the no-slip BC has since been used quite successfully to describe macroscopic flows [8,9]. In the past decades, the fast development of micro- and nanofluidics [10-13] has pushed the community to reconsider the no-slip BC. Both experimental and theoretical studies have shown that liquids can slip on solid surfaces and the slip is well described by the Navier BC [2]. It is of critical importance to understand and optimize liquid-solid slip, which can enhance dramatically the performance of micro- and nanofluidic systems [14-17]. Accordingly, the hydrodynamic BC has been an active field of research during the past decades, both on the experimental side [18-21] and on the modeling side [3,4,6,22-33], where molecular dynamics (MD) simulation proved to be a powerful tool. However, determining both $\lambda$ and $z_{s}$ that make up the Navier BC is delicate even in a MD simulation, and it is a common practice to assume that $z_{s}$ lies at some prescribed distance from the wall surface [32] or coincides with the position of the wall surface [23-25] and only the friction coefficient (or equivalently the slip length) is measured. Few groups have attempted the complete determination of the Navier BC so far: for instance, Bocquet and Barrat [3] reported the gap $\Delta$ between the wall surface and the hydrodynamic wall surface (hydrodynamic boundary) was $1.3 \sigma$ by a Green-Kubotype formula and $1.6 \sigma$ as a fit parameter to reproduce the measured autocorrelation function of the fluctuating liquid velocity confined between two walls, where $\sigma$ is the liquid molecular diameter; Chen et al. [4] measured $\Delta$ between 2.2 and $2.5 \sigma$ from an analysis of the equilibrium hydrodynamic fluctuations; and recently Herrero et al. [34] measured $\Delta$ varying between 0.6 and $1.1 \sigma$ depending on the wall wettability from the analysis of the wall shear stress in Poiseuille flow simulations. Camargo et al. [6] proposed an alternative derivation of the Navier BC without resorting to the linear response theory, including the definition of $\Delta$.

About the liquid-solid FC $\lambda$, it has generally been assumed to exhibit a pure viscous behavior following Navier's initial assumption. Recently, however, Cross et al. [5] emphasized that fluids are in general viscoelastic [35], and that in the Navier BC both the shear viscosity and the FC can be complex quantities. Using a dynamic surface force apparatus, they characterized the response of a viscoelastic aqueous solution of polymers periodically squeezed between a sphere and a plane. They showed that the shear viscosity indeed displayed a viscoelastic behavior, but they could not detect any elastic component in the interfacial friction. They discussed that the simple Newtonianlike FC reflected the dynamics of an interfacial depletion layer made of pure water, and suggested that it would be interesting to perform experiments on interfaces designed to exhibit viscoelastic friction. There have also been experimental works on the interfacial rheology by means of quartz crystal microbalance (QCM) [10,36-38], where the bulk viscoelasticity of the liquid film and the Newtonian-like slip on the wall surface were assumed to model the measured shift of the resonance frequency of the crystal. On the numerical side, Huang and Szlufarska [27] calculated the frequency dependence of the complex FC through a frequency analysis of the fluctuating friction force on a model Lennard-Jones (LJ) fluid by means of equilibrium MD simulation. Priezjev [39] investigated the Newtonian-like FC of a LJ liquid in slowly oscillating Couette flows by nonequilibrium MD simulation and analyzed the connection between the FC and the static structure factor of the first liquid adsorption layer under the unsteady motion.

In the present study, we show that by combining continuum mechanics and nonequilibrium MD simulation, the hydrodynamic boundary condition can be fully characterized, where both the complex FC and the hydrodynamic boundary position were identified. For the system to be investigated, we chose a generic LJ liquid confined between parallel walls and analyzed the frequency response of the liquid to the tangentially oscillating motion of the walls. 


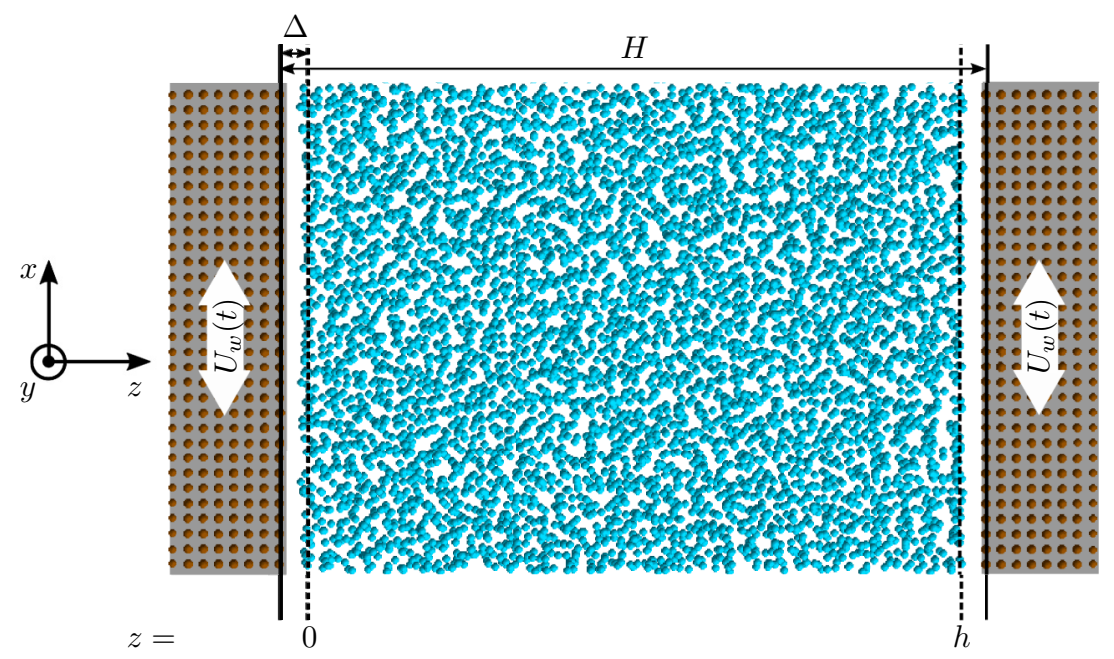

FIG. 1. Schematic of the considered system. The positions of the liquid molecules (light blue) and the solid atoms that compose the walls (orange) were taken from a snapshot of an MD simulation result described in Sec. III. The domain boundaries normal to the $x$ and $y$ axes are connected with the periodic BC and therefore the effective liquid velocity is only a function of $z$ and $t$.

\section{THEORY}

In the present study, we attempt to identify the BC imposed by the wall on the bulk liquid motion in nanometric systems, where the slip length cannot be neglected. Figure 1 shows the schematic and the coordinates of the system considered in the present study. A liquid is confined between two parallel solid walls that translate together in the wall-tangential $x$ direction with a time-dependent velocity $U_{w}(t)$. In general, the wall surface is different from the hydrodynamic boundary, due to the inhomogeneous liquid density distribution in the atomic vicinity of the wall. We define the hydrodynamic wall positions as $z=0$ and $h$. Because the Reynolds number is low in the flows investigated in the present study, we suppose that the motion of the liquid in the bulk region follows the Stokes equation

$$
\frac{\partial u(z, t)}{\partial t}=\frac{\eta}{\rho} \frac{\partial^{2} u(z, t)}{\partial z^{2}}
$$

under the Navier BC defined on the hydrodynamic boundaries

$$
\begin{aligned}
& \left.\eta \frac{\partial u(z, t)}{\partial z}\right|_{z=0}=\int_{0}^{t} \lambda\left(t-t^{\prime}\right)\left[u\left(0, t^{\prime}\right)-U_{w}\left(t^{\prime}\right)\right] d t^{\prime} \equiv \tau_{w} \\
& \left.\eta \frac{\partial u(z, t)}{\partial z}\right|_{z=h}=\int_{0}^{t} \lambda\left(t-t^{\prime}\right)\left[U_{w}\left(t^{\prime}\right)-u\left(h, t^{\prime}\right)\right] d t^{\prime}=-\tau_{w}
\end{aligned}
$$

where $u, t, \rho, \eta, \lambda$, and $\tau_{w}$ denote the liquid velocity in the $x$ direction, the time, the bulk liquid density, the bulk liquid viscosity, the frequency-dependent liquid-solid FC, and the wall shear stress, respectively. Note that the velocity $u$ is the effective liquid velocity that describes the bulk liquid motion and in the close vicinity of the wall $u$ may deviate from a locally measured liquid velocity. To model the viscoelastic FC, we write the frequency dependence of $\lambda$ as

$$
\tilde{\lambda}(\omega)=\frac{\lambda_{0}}{1+i \omega t_{\lambda}}
$$


where $\omega$ is the angular frequency and the tilde denotes that the variable is Fourier-Laplace transformed. Equation (3) is an analog to the Maxwell model of viscosity with a relaxation time $t_{\lambda}$. The friction kernel $\tilde{\lambda}$ has the following asymptotic behaviors: $\tilde{\lambda} \simeq \lambda_{0}$ when $\omega t_{\lambda} \ll 1$ while $\tilde{\lambda} \simeq \lambda_{0} /\left(i \omega t_{\lambda}\right)$ when $\omega t_{\lambda} \gg 1$. This latter behavior is consistent with the principle of causality which implies that $\lambda(t)$ is a step function near $t=0$. In principle, the liquid viscosity $\eta$ can also be frequency dependent in a similar way, but as we see and discuss in Sec. IV A, the present system is well described by a frequency-independent Newtonian viscosity. We assume that the Navier BC, given by Eqs. (2) and (3) with constants $\lambda_{0}, t_{\lambda}$, and $h$, is valid for the whole frequency range, which is further addressed in the Appendix.

From the solution of Eqs. (1) and (2), the response function of the wall shear stress $\tau_{w}$ to the wall velocity $U_{w}, \tilde{Z}_{\tau} \equiv-\tilde{\tau}_{w} / \tilde{U}_{w}$, is obtained:

$$
\tilde{Z}_{\tau}=\tilde{\lambda} \frac{-\tilde{b} \zeta(1-\cosh (\zeta h))+\tilde{b}^{2} \zeta^{2} \sinh (\zeta h)}{2 \tilde{b} \zeta \cosh (\zeta h)+\left(\tilde{b}^{2} \zeta^{2}+1\right) \sinh (\zeta h)} \quad \text { with } \zeta=\sqrt{i \rho \omega / \eta}
$$

where $\tilde{b}(\equiv \eta / \tilde{\lambda})$ is the complex slip length. Because $\tilde{Z}_{\tau}$ is directly measurable, we can obtain the unknown quantities $\lambda_{0}, t_{\lambda}$, and $h$ as the fitting parameters to the measured results. In addition to Eq. (4), we can write the response function of the mean velocity $\bar{u}\left(=(1 / h) \int_{0}^{h} u(z, t) d z\right)$ to the wall velocity $U_{w}, \tilde{Z}_{\bar{u}} \equiv \tilde{\bar{u}} / \tilde{U}_{w}$, as

$$
\tilde{Z}_{\bar{u}}=\frac{2}{\zeta h} \frac{\cosh (\zeta h)+\tilde{b} \zeta \sinh (\zeta h)-1}{2 \tilde{b} \zeta \cosh (\zeta h)+\left(\tilde{b}^{2} \zeta^{2}+1\right) \sinh (\zeta h)} \quad \text { with } \zeta=\sqrt{i \rho \omega / \eta},
$$

which is useful to see the frequency-dependent flow rate of the system. The shear stress and velocity response have the following asymptotic behaviors: $\tilde{Z}_{\tau} \simeq \eta h \zeta^{2} / 2=i \rho h \omega / 2$ and $\tilde{Z}_{\bar{u}} \simeq 1$ when $\omega t_{\lambda} \ll 1$ and $\tilde{Z}_{\tau} \simeq \tilde{\lambda}$ and $\tilde{Z}_{\bar{u}} \simeq 2 /\left(\tilde{b} h \zeta^{2}\right)$ when $\omega t_{\lambda} \gg 1$, where $\tilde{\lambda} \simeq \lambda_{0} /\left(i \omega t_{\lambda}\right)$ and $\tilde{b} \simeq i \omega \eta t_{\lambda} / \lambda_{0}$.

\section{MOLECULAR DYNAMICS SIMULATIONS}

To measure the response of the wall shear stress to the wall velocity $\tilde{Z}_{\tau}$, we performed nonequilibrium MD simulations. As schematically shown in Fig. 1, the investigated system was a LJ liquid confined between two parallel nonpolar walls. We considered 6400 molecules for the liquid and 8192 atoms for the walls. All the quantities in the present study are shown in LJ reduced units based on the liquid molecular mass $m_{f}$ and two parameters $\sigma_{f f}$ and $\varepsilon_{f f}$ for the LJ potential $\Phi_{\mathrm{LJ}}\left(r_{i j}\right)=4 \varepsilon\left[\left(\sigma / r_{i j}\right)^{12}-\left(\sigma / r_{i j}\right)^{6}\right]$ between the liquid molecules, where $r_{i j}$ was the distance between molecules $i$ and $j$. We added a quadratic function to $\Phi_{\mathrm{LJ}}$ so that $\Phi_{\mathrm{LJ}}\left(r_{i j}\right)$ and $\Phi_{\mathrm{LJ}}^{\prime}\left(r_{i j}\right)$ smoothly vanished at the cutoff distance $r_{i j}=3.50$. Each solid wall consisted of eight layers of atoms in the (001) plane of a fcc crystal with a lattice constant of 1.15; therefore, the wall surface was atomically smooth. The interaction between the wall atoms was described by the harmonic potential between the nearest neighbors $\Phi_{\mathrm{H}}\left(r_{i j}\right)=k\left(r_{i j}-r_{0}\right)^{2} / 2$ with $k=3.24 \times 10^{3}$ and $r_{0}=0.815$. For the liquid-solid interaction, the $\mathrm{LJ}$ potential was adopted. To investigate three different wall wettabilities to the liquid, three different $\varepsilon_{f w}$ were used while $\sigma_{f w}=1.01$ was kept constant as shown in Fig. 2. Wall $\mathrm{C}$ was perfectly wetted by the liquid and wall $\mathrm{A}$ was the least wetted by the liquid, where an equilibrium liquid droplet formed a contact angle of $140^{\circ}$ under the same temperature as the present study.

The equation of motion for each atom was integrated by means of the velocity Verlet algorithm with a time step of $2.33 \times 10^{-3}$. The temperature of the system was controlled at 0.827 by applying a Langevin thermostat to the solid atoms in the second outermost layer of each wall. The thermostatting layer was placed far enough from the liquid so that the thermostatted wall atoms did not directly interact with the liquid molecules. We limited the temperature control to the velocities of the thermostatted wall atoms in the $x-y$ plane to avoid the fluctuation in the system pressure as a possible side effect [40]. The height of the channel, $H$, was determined before the main calculation so that the system pressure was 0.094 and the bulk density $\rho$ was 0.76 : a channel with a different 


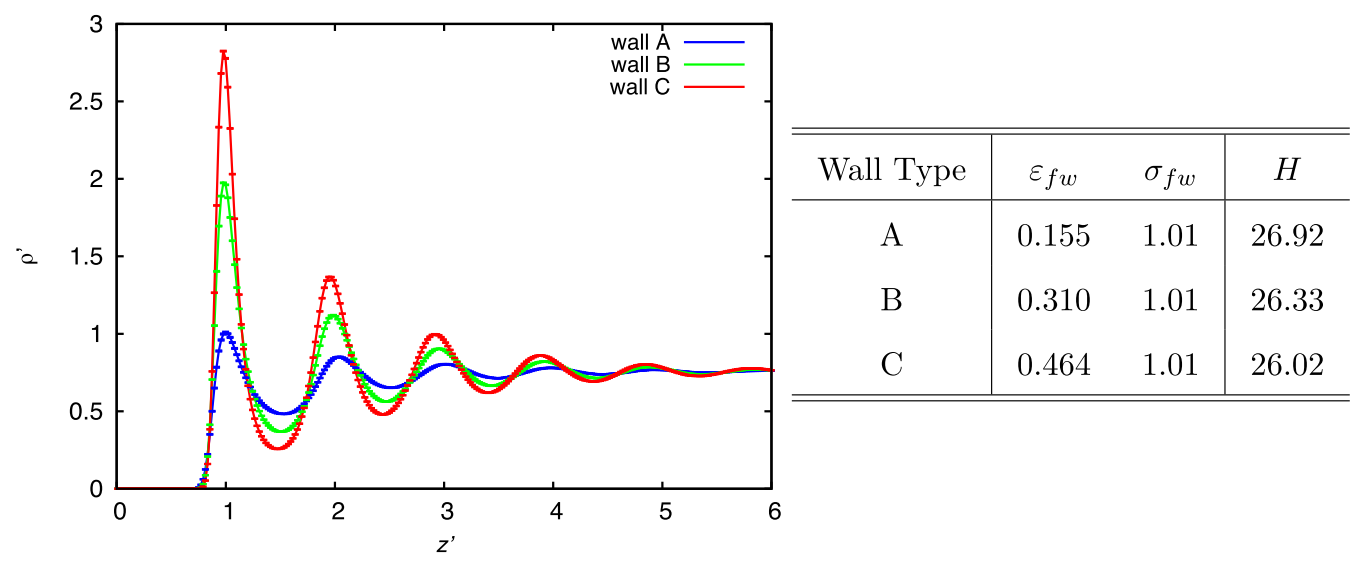

FIG. 2. Left: Distribution of the local liquid density $\rho^{\prime}$ near the three walls investigated in the present study. The origin of the coordinate $z^{\prime}$ is on the innermost (001) plane of the left fcc crystal depicted in Fig. 1. Right: The parameters for the liquid-solid interaction potential and the channel height for each wall.

wall type had a different channel height as shown in the table of Fig. 2. The bulk shear viscosity $\eta$ and the kinematic viscosity $\eta / \rho$ of the liquid were 1.66 and 2.18 , respectively. We confirmed that there was no system size effect by comparing the present results to the simulation results with 3840 liquid molecules with the confining walls being unchanged.

We measured the wall shear stress $\tau_{w}$ under the prescribed wall velocity $U_{w}(t)=U_{0} \sin (\omega t)$ with $U_{0}=0.063$ and various angular frequencies $\omega$. Doubling $U_{0}$ did not affect the results and therefore the system was assumed to be in the linear response regime. To perform a Fourier-Laplace transform of a time-dependent quantity $g(t)$ measured in our numerical experiments, we truncated $g(t)$ at a finite time $T$, and

$$
W(t) g(t) \quad \text { with } W(t)= \begin{cases}1 & (0 \leqslant t \leqslant T) \\ 0 & (t>T)\end{cases}
$$

was considered to be the integrand of the Fourier-Laplace transform, where $T=n \pi / \omega$ with an integer $n$ was chosen to ensure the mutual orthogonality of the base functions. The upper time limit $T$ was about $7 \times 10^{4}$ in the present study. By virtue of Eq. (4), we obtained the three unknown parameters to describe the system, namely, $\lambda_{0}, t_{\lambda}$, and $h$ to achieve the least square to $\sum_{\omega}\left[\tilde{Z}_{\tau}\left(\omega ; \lambda_{0}, h, t_{\lambda}\right)+\tilde{\tau}_{w} / \tilde{U}_{w}\right]^{2}$. The nonlinear least-squares problem was solved by the Levenberg-Marquardt method. The liquid viscosity $\eta$, which was treated as a constant, was obtained independently in a Couette flow MD simulation under identical temperature and pressure as the present study, as the ratio between the shear stress and the bulk shear rate.

For all results in the present study we show the statistical uncertainties although in some figures the error bars are indiscernibly small. For simple time-averaged quantities, we show the $95 \%$ confidence interval of the average that we calculated from the independent samples extracted from the time-series data, unless otherwise stated. To estimate the statistical uncertainties of the parameters for the least-squares fits, we adopted the method described by Berendsen [41] involving the inverse of the data error matrix.

\section{RESULTS AND DISCUSSION}

\section{A. Identification of the hydrodynamic boundary condition}

As described in Sec. III, the frequency-dependent FC and the position of the hydrodynamic boundary were identified as the parameters to give the best fit to the response function of the wall shear stress to the wall velocity obtained by the MD simulations: the solid lines in the left panel of 

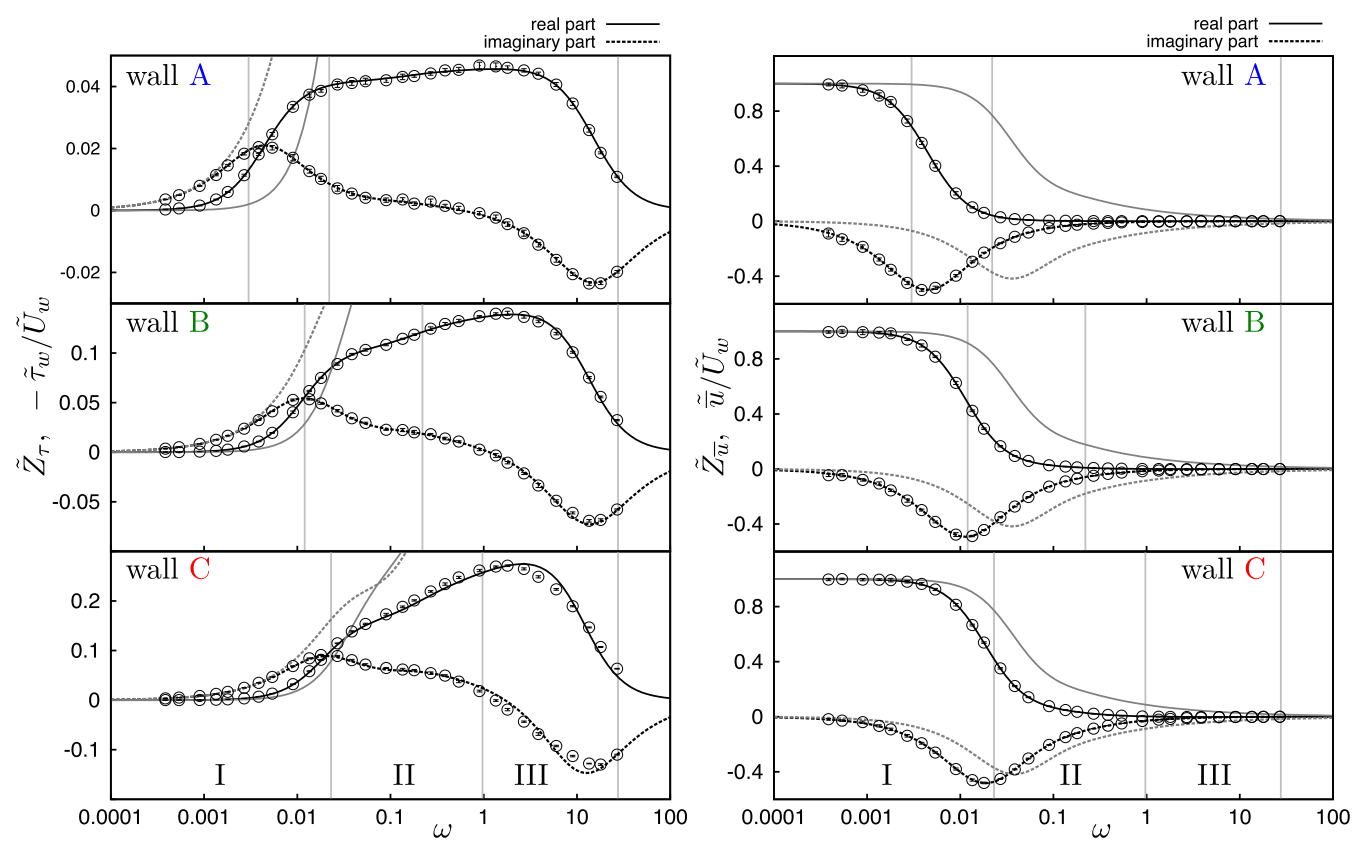

FIG. 3. Response functions for walls A, B, and C. Symbols show results obtained by MD simulations (left, $-\tilde{\tau}_{w} / \tilde{U}_{w}$; right, $\tilde{\bar{u}} / \tilde{U}_{w}$ ) and black solid and dotted lines show the fitting curves to the MD results (left, $\tilde{Z}_{\tau}$; right, $\tilde{Z}_{\bar{u}}$ ). The vertical gray lines indicate the boundaries between frequency regimes classified based on the comparison of the penetration length $2 \sqrt{\pi \eta /(\rho \omega)}$ to the characteristic lengths in the system. Predictions assuming the no-slip boundary condition are shown with dark gray lines for comparison. The error bars, which are smaller than the symbols, show the $95 \%$ confidence interval estimated from seven independent simulation runs. One simulation run was made of 4 oscillation cycles for the lowest frequency and $3 \times 10^{5}$ cycles for the highest frequency.

Fig. 3 show the result of the fit and Table I summarizes the parameters to construct the fit. The period of the fastest wall oscillation in the present study was three times larger than the decorrelation times of the solid-liquid and liquid-liquid forces, which were 0.08 and 0.07 , respectively. The error bars in Fig. 3 show the $95 \%$ confidence interval estimated from seven independent simulation runs. One simulation run was made of 4 oscillation cycles for the lowest frequency and $3 \times 10^{5}$ cycles for the highest frequency. The data error matrix to estimate the statistical uncertainties shown in Table I was built from the measurement uncertainties in the frequency response of the wall shear stress shown in the left panel of Fig. 3.

We see that the fit based on Eq. (4) with the frequency-dependent viscoelastic FC, Eq. (3), is excellent over the whole range of frequency regardless of the liquid wettability to the wall. It is interesting to observe that in the zero-frequency limit the wall shear stress response $\tilde{Z}_{\tau}$ asymptotes to zero and the mean velocity response $\tilde{Z}_{\bar{u}}$ asymptotes to 1 , which is in accordance with the asymptotic

TABLE I. Three parameters to form the Navier BC on the hydrodynamic boundary, which was obtained to give the best fit in the left panel of Fig. 3 .

\begin{tabular}{lccc}
\hline \hline Wall type & $\lambda_{0}$ & $t_{\lambda}$ & $\Delta$ \\
\hline A & $0.0470 \pm 0.0001$ & $0.067 \pm 0.001$ & $1.02 \pm 0.06$ \\
B & $0.1492 \pm 0.0003$ & $0.077 \pm 0.001$ & $0.73 \pm 0.04$ \\
C & $0.311 \pm 0.001$ & $0.0894 \pm 0.0004$ & $0.90 \pm 0.03$ \\
\hline \hline
\end{tabular}


behaviors derived in Sec. II. It is because the boundary layer is fully developed and there is no friction between the liquid and the wall in this limit. The zero-frequency component of the FC $\lambda_{0}$ increased when the wall wettability was increased, following a general tendency rationalized previously [23]. The relaxation time $t_{\lambda}$ of the FC was slightly larger for larger wettability between liquid and solid. The gap between the wall surface and the hydrodynamic boundary was calculated as $\Delta=(H-h) / 2$. The small variations in $\Delta(<0.3)$ observed among the different wall types did not show a consistent dependence on the wall wettability and can also be frequency dependent (see the Appendix). The positions of the hydrodynamic boundary were all in the first adsorption layer: fixing $\Delta=1.00$ did not bring significant changes in the other two fit parameters but it made the error in the fit larger than the present three-parameter fit. The measured values of $\Delta$ were somewhat smaller than those reported by Bocquet and Barrat [3] and about half of those reported by Chen et al. [4], but the direct comparison is not possible because of the difference in the system, especially in the liquid-solid interaction potential. There is also a subtle conceptual difference between the present study and the studies by Bocquet and Barrat [3] and Chen et al. [4]: they assumed the velocity on the hydrodynamic boundary was the averaged liquid velocity on that location, but we considered the liquid velocity on the hydrodynamic boundary was the effective velocity that gave the wall shear stress through the Navier BC, Eq. (2). The right panel of Fig. 3 shows the response function of the mean velocity to the wall velocity, where we compare the MD simulation results and the analytical solution, Eq. (5), with the parameters shown in Table I. We see that Eq. (5), which was derived from the Stokes equation with the Navier slip BC, describes well the MD simulation results over the whole frequency range.

We can classify the investigated wall frequency range into three regimes according to the magnitude of the penetration length $2 \sqrt{\pi \eta /(\rho \omega)}$ in comparison to the characteristic lengths in the system. These characteristic lengths $l$ are $H+2 b_{0}-2 \Delta$ with $b_{0} \equiv \eta / \lambda_{0}$, which is the effective channel height; $b_{0}$, which is the slip length; and $\sigma$, which is the height of the first adsorption layer from the wall surface, and they define the boundaries between the frequency regimes shown as vertical gray lines in Fig. 3 from left to right by solving $l=2 \sqrt{\pi \eta /(\rho \omega)}$ for $\omega$. In regime I the flow in the channel can be considered close to uniform and in regime III the flow in the channel can be neglected. Regime II represents the flow regime between the two regimes I and III. These flow characteristics of each regime can be observed in the velocity profiles shown in Fig. 4.

In the high-frequency range, such as $\omega>1$, the elastic component of the liquid-solid friction was important according to the value of $t_{\lambda}$ while the magnitude of the friction was still significant. In this high-frequency range, which belongs to regime III, there was almost no mean flow in the channel and thus the effect of the bulk shear viscosity on the interfacial behavior of the system was negligible. It justifies to neglect the elastic component of the bulk shear viscosity in the present study, considering that the Maxwell relaxation time of the bulk shear viscosity $[42,43]$ is at most comparable to $t_{\lambda}$. The frequency range $\omega>1$ corresponds to the gigahertz range for a simple liquid (for argon $\omega=1$ means $74.4 \mathrm{GHz}$ ), which can be reached by a recent nanoultrasonic measurement technique [44] to probe the interfacial rheology.

The two panels of Fig. 3 illustrate the difficulty to obtain the liquid-solid FC through the evaluation of the Green-Kubo integral of the friction force autocorrelation function [3,45] given by equilibrium MD simulations. In the evaluation of the Green-Kubo integral in systems of finite size, it is often assumed that the mass of the fluid involved in the liquid-solid friction is a finite constant [46-48], but the present results show that it is actually frequency dependent.

In Fig. 3, for comparison, the predictions assuming no slip on the hydrodynamic boundaries are shown with dark gray lines, where the response functions can be written respectively as

$$
\tilde{Z}_{\tau}^{\text {no-slip }}=\frac{\eta \zeta(\cosh (\zeta h)-1)}{\sinh (\zeta h)}
$$

and

$$
\tilde{Z}_{\bar{u}}^{\text {no-slip }}=\frac{2(\cosh (\zeta h)-1)}{\zeta h \sinh (\zeta h)}
$$




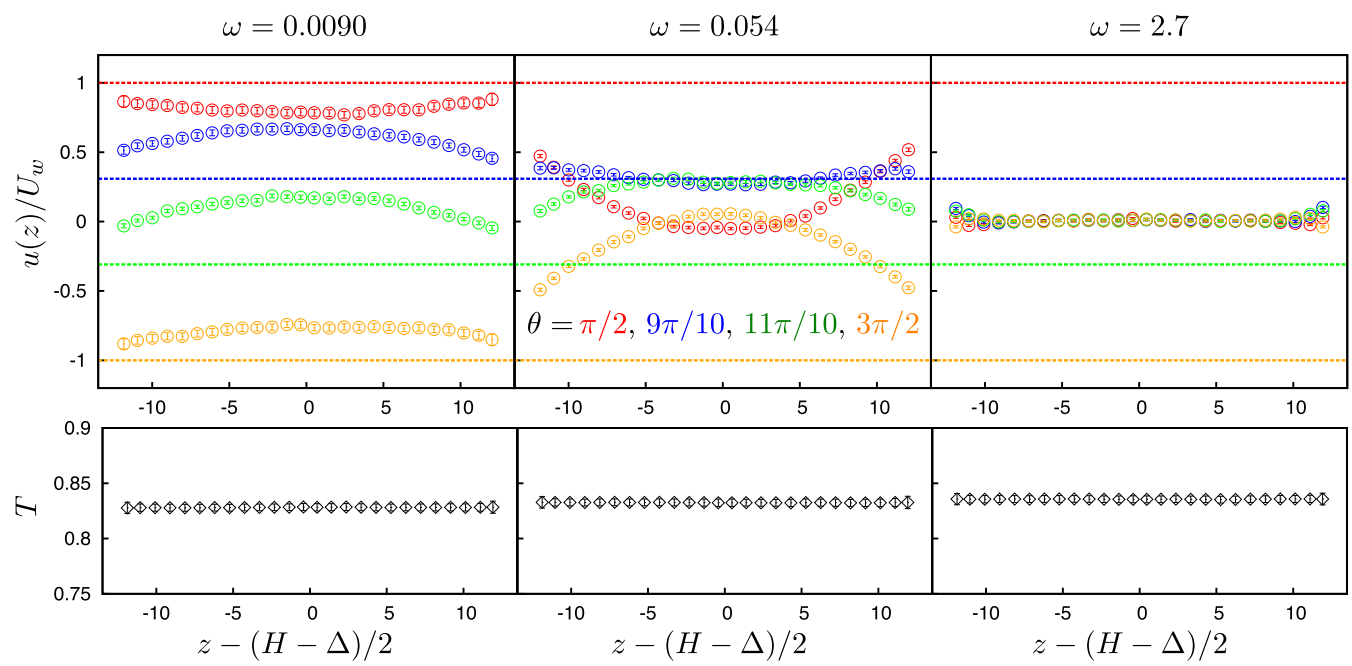

FIG. 4. Velocity and temperature distributions in the channel (wall C) for three wall oscillation frequencies $\omega$ that belong to regimes I, II, and III, respectively. The velocity distributions (symbols) and the wall velocities (dotted lines) at four phases $(\theta=\pi / 2,9 \pi / 10,11 \pi / 10$, and $3 \pi / 2)$ are shown. The temperature was almost uniform in the channel and well controlled around the target temperature. The data were averaged over 100 , 600 , and 30000 oscillation cycles for $\omega=0.0090,0.054$, and 2.7, respectively, which was the same condition to obtain the corresponding data plots in Fig. 3. Most of the error bars are smaller than the symbols.

both with $\zeta=\sqrt{i \rho \omega / \eta}$. The no-slip boundary condition clearly fails to predict the flow in the present systems, although it shows better agreement with the MD simulation results for the more wettable walls as expected.

\section{B. Application of the hydrodynamic boundary condition}

In Sec. IV A we identified the hydrodynamic BC to describe the bulk motion of the liquid. In this section we see if the BC works for the two most fundamental flow problems, a Couette flow, which is wall driven, and a Poiseuille flow, which is pressure driven. These flows are steady flows and therefore we only consider the zero-frequency component of the FC $\lambda_{0}$ here. According to Priezjev [39], the identical $\lambda_{0}$ can be employed for oscillatory and steady shear flows.

Figure 5 compares the liquid velocity distributions given by the continuum predictions and MD simulation results. In the MD simulations, each local liquid velocity was obtained as the average in
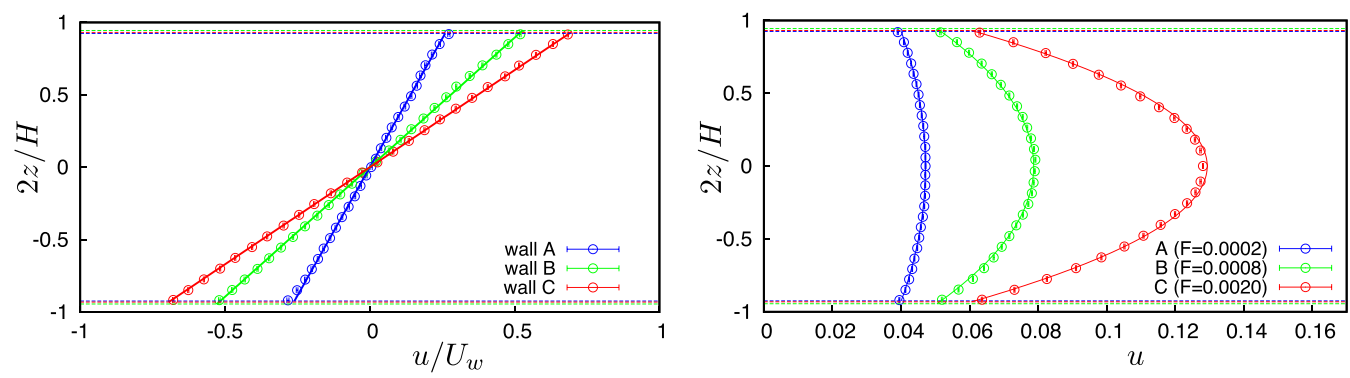

FIG. 5. Comparison of the velocity profiles given by the Stokes equation with the Navier BC (solid lines) and by the MD simulations (symbols). The origin of the $z$ axis is on the centerline of the channel and the dotted lines show the position of the hydrodynamic boundary for each wall. The error bars are smaller than the symbols. 

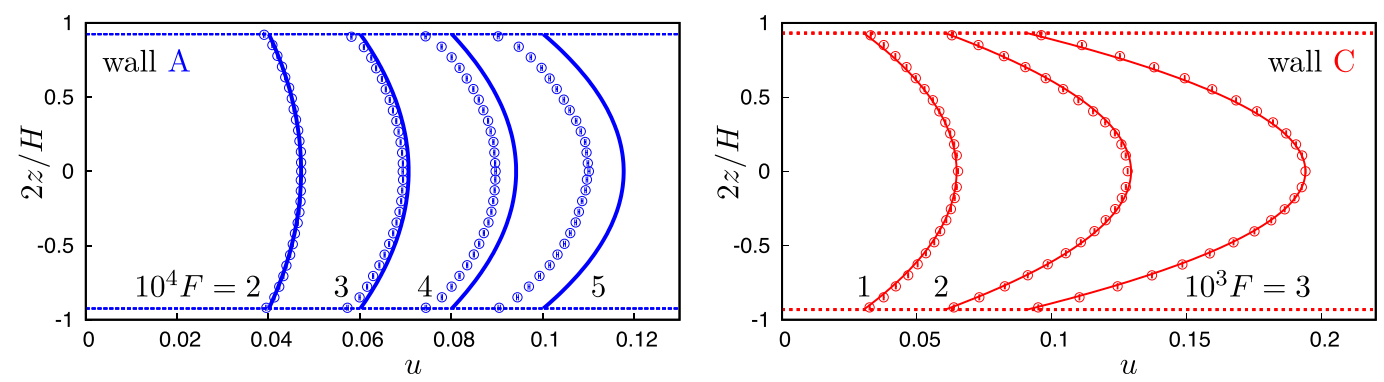

FIG. 6. The velocity profile of pressure-driven flows confined between walls A (left panel) and C (right panel), given by the Stokes equation and the Navier BC (solid lines) and by the MD simulation (symbols). The configurations are the same as in Fig. 5. The error bars are smaller than the symbols.

a bin with a height of 1 , where the same liquid and wall as in Sec. IV A were used and so was the temperature and pressure control of the system. The origin of the $z$ axis was set on the centerline of the channel and the position of the hydrodynamic boundary was therefore $z= \pm h / 2$. The left panel of the figure shows results for the Couette flow problem, where the top and bottom walls translated with a constant velocity $U_{w}$ and $-U_{w}$, respectively. The predicted liquid velocity is written as

$$
u(z)=\frac{2 z}{h+2 b_{0}} U_{w}
$$

where $b_{0}$ is the slip length $\eta / \lambda_{0}$. For all wall cases, the bulk liquid velocity was well predicted by the present hydrodynamic $\mathrm{BC}$. For the least wettable wall case, the velocity profile showed a small deviation from the linear profile in the first adsorption layer, which can be attributed to the local viscosity variation [49,50]. The right panel of the figure shows results for the Poiseuille flow problem. The predicted liquid velocity is written as

$$
u(z)=\frac{\rho f}{2 \eta}\left(\frac{h^{2}}{4}+h b-z^{2}\right),
$$

where an acceleration $f$ per unit mass of the bulk liquid is assumed. In the MD simulation, the external force per liquid molecule $F$ was given by $F=\rho f L_{x} L_{y} h / N$, where $L_{x}$ and $L_{y}$ denote the domain sizes in $x$ and $y$ directions and $N$ is the number of liquid molecules [51]. For walls B and $\mathrm{C}$, the liquid velocity in the bulk region was well predicted with the present $\mathrm{BC}$. Only for wall A, i.e., the least wettable wall, did the MD simulation results deviate from the continuum predictions when we imposed stronger acceleration as shown in Fig. 6. The figure shows that the FC on the least wettable wall becomes nonlinear for the slip velocity where the FC on the other walls stays in the linear response regime. The figure also shows that the FC becomes larger in the nonlinear regime, which was also found by Alizadeh Pahlavan and Freund [52] for a similar system as the present one, without direct thermostatting of the liquid.

\section{CONCLUSION}

In this article, we derived analytically the frequency-dependent response of a nanofluidic slab to parallel oscillations of the confining walls as a solution of the Stokes equation with the Navier slip BC. Specifically, we derived two response functions quantifying the wall shear stress and the mean liquid velocity versus the wall velocity. The response functions were expressed as a function with the parameters characterizing the hydrodynamic boundary condition at the walls. A viscoelastic friction was considered, quantified by the zero-frequency friction coefficient $\lambda_{0}$, the viscoelastic relaxation time $t_{\lambda}$, and the effective hydrodynamic height of the slab, $h$. 
We then performed MD simulations of a generic LJ liquid confined between parallel nonpolar walls of three different wettabilities, and measured the nonequilibrium liquid response to oscillations of the walls at different frequencies. The analytical formulas for the shear stress and mean velocity responses constructed excellent fitting curves to the simulation results for the three wall wettabilities we considered. From the fitting, we could extract $\lambda_{0}, t_{\lambda}$, and $h$, hence fully characterizing the frequency-dependent hydrodynamic boundary condition at the walls.

We found that the zero-frequency FC increased with increasing wall wettability, which was rationalized previously in the literature. We quantified the viscoelastic relaxation time for the liquid-solid interface, which increased weakly with wettability, and was on the order of the Maxwell relaxation time for the shear viscosity of a bulk LJ liquid reported in the literature. Importantly, we characterized the hydrodynamic position of the wall surface, which has been little explored in the literature. We found that it lies at a distance within one liquid molecular diameter inside the liquid from the wall surface. The position varied slightly and inconsistently with the wall wettability and can also be frequency dependent (see the Appendix). The oscillating-wall simulations have also shown that the system's response is only significant in a given frequency range, which can help in understanding and refining equilibrium measurements of interfacial friction using the Green-Kubo formalism. We also hope that the present analysis is useful in the development of the theoretical models that the measurements such as QCM rely on, especially in nanoconfined environments.

Finally, we confirmed that the hydrodynamic BC measured via the oscillating-wall method predicted accurately both Couette and Poiseuille flows in the same slab geometry, in the linear response regime. Overall the oscillating-wall method can be used to characterize extensively the hydrodynamic boundary condition as it provides information on the viscoelastic friction coefficient $\lambda$, the hydrodynamic wall surface position $z_{s}$, and their frequency dependencies. We emphasize that the knowledge of both $\lambda$ and $z_{s}$ is required to fully quantify the hydrodynamic BC, and to predict flows in nanochannels. We hope the proposed method will be useful to characterize more complex liquid-solid interfaces of interest under a variety of thermodynamic conditions, in particular for flows of complex fluids or fluids at a low temperature. Information on the viscoelastic interfacial behavior should also be useful for methodological developments related to the characterization of the liquid-solid slip.

\section{ACKNOWLEDGMENTS}

T.O. is grateful to Prof. Takeo Kajishima of Osaka University for the continuous encouragement to carry out this work. The authors thank Kosuke Tatsuta and Hiroki Kusudo of Osaka University for providing the numerical code for the Levenberg-Marquardt method and the contact angle data, respectively. This work was financially supported by JSPS KAKENHI Grants No. 18K03929 and No. 18K03978. Y.Y. was also supported by JST CREST Grant No. JPMJCR18I1, Japan. L.J. was supported by the ANR, Project No. ANR-16-CE06-0004-01 NECtAR and the Institut Universitaire de France. L.J. benefited from a JSPS international fellowship for research in Japan.

\section{APPENDIX: FREQUENCY DEPENDENCE OF THE HYDRODYNAMIC BOUNDARY}

In the present study, we assumed that the coefficient $\lambda_{0}$ in the liquid-solid FC (called the zerofrequency component of the FC) and the position of the hydrodynamic boundary $\Delta$ (equivalently the hydrodynamic height of the system, $h$ ) are independent of the oscillating wall frequency. Here we show how the frequency range used to fit the curves in the left panel of Fig. 3 affects the estimation of $\lambda_{0}$ and $h$.

Since we know the relaxation time $t_{\lambda}$ of the FC from the observations in Sec. IV A, we can estimate the frequency $\omega$ below which the elastic component of the FC is negligible. For $\omega \leqslant 0.54$, the elastic contribution to the magnitude of the complex FC should be less than $5 \%$ for all wall types investigated in the present study. Figure 7 shows how the two fitting parameters $\lambda_{0}$ and $h$ (converted to $\Delta$ by $\Delta=(H-h) / 2$ ) depend on the number of data points $n_{\omega}$ below $\omega=0.54$ to construct the 

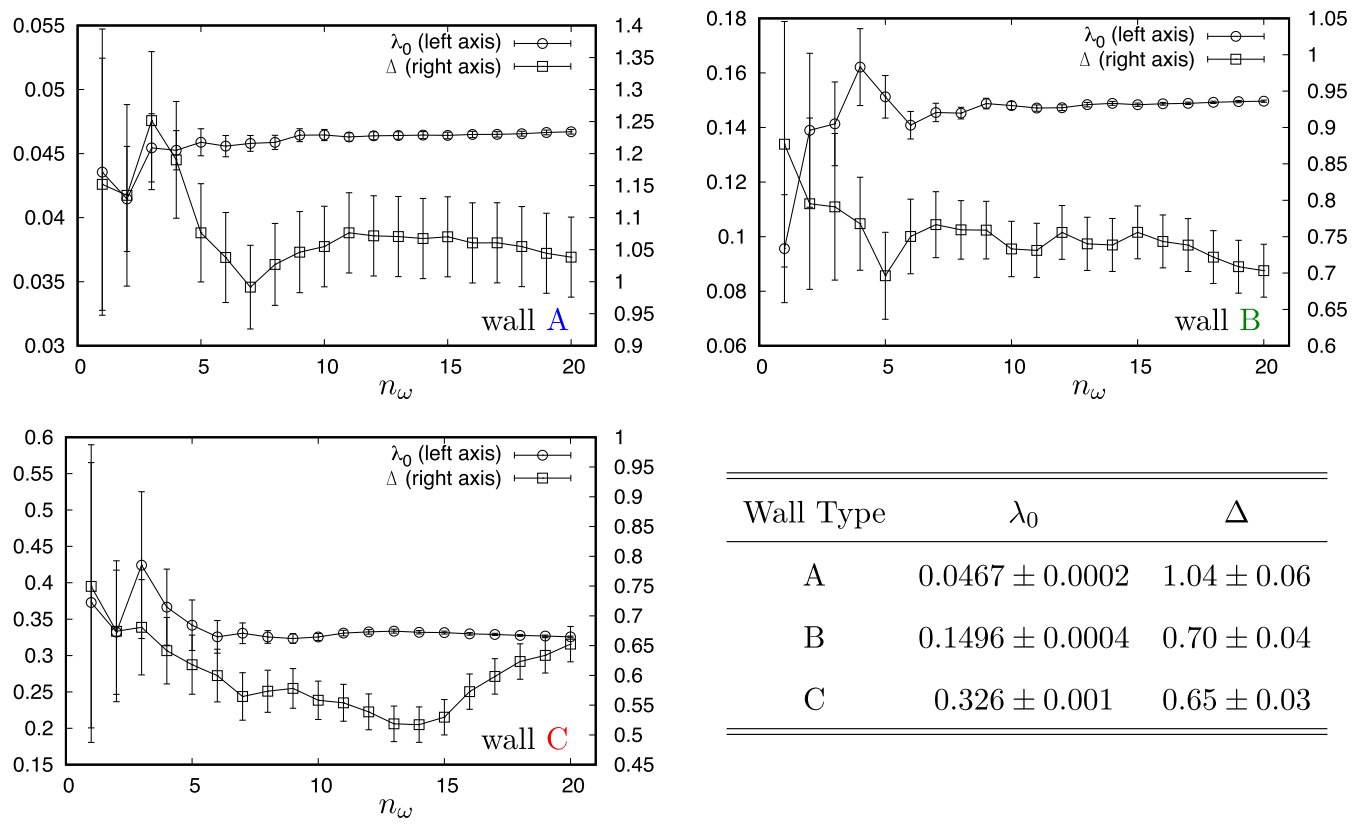

\begin{tabular}{ccc}
\hline \hline Wall Type & $\lambda_{0}$ & $\Delta$ \\
\hline A & $0.0467 \pm 0.0002$ & $1.04 \pm 0.06$ \\
B & $0.1496 \pm 0.0004$ & $0.70 \pm 0.04$ \\
C & $0.326 \pm 0.001$ & $0.65 \pm 0.03$ \\
\hline \hline
\end{tabular}

FIG. 7. Dependence of the fit parameters in Eq. (4), the zero-frequency component of the FC $\lambda_{0}$, and the hydrodynamic system height shown in terms of $\Delta$, on the frequency range below 0.54 to construct the fit. The table shows the fit parameters when the number of data points $n_{\omega}=20$, which corresponds to the frequency range $0<\omega \leqslant 0.54$.

least-squares fit to the data $-\tilde{\tau}_{w} / \tilde{U}_{w}$. While $\lambda_{0}$ seems well converged with $n_{\omega}=20$ for all walls, the convergence of $\Delta$ is not reached for wall $\mathrm{C}$. In fact, the value of $\Delta$ obtained here for wall $\mathrm{C}$ is much smaller than the value obtained to fit the data over the whole frequency range up to $\omega=27$ (Table I), which indicates that the position of the hydrodynamic boundary is frequency dependent and might explain the small discrepancy between the analytical prediction and the MD simulation result for wall $\mathrm{C}$ in Fig. 3. Figure 7 also tells that the value of $\Delta$ in the pure viscous regime is smaller for a wall with larger wettability. It is reminiscent of the recent work by Herrero et al. [34], which theoretically demonstrated this $\Delta$ dependence on the wall wettability for a steady channel flow driven by a gravitational acceleration.

[1] C.-L. Navier, Memoire sur les lois du mouvement des fluides, Mem. Acad. Sci. Inst. Fr. 6, 389 (1823).

[2] L. Bocquet and J.-L. Barrat, Flow boundary conditions from nano- to micro-scales, Soft Matter 3, 685 (2007).

[3] L. Bocquet and J.-1. Barrat, Hydrodynamic boundary conditions, correlation functions, and Kubo relations for confined fluids, Phys. Rev. E 49, 3079 (1994).

[4] S. Chen, H. Wang, T. Qian, and P. Sheng, Determining hydrodynamic boundary conditions from equilibrium fluctuations, Phys. Rev. E 92, 043007 (2015).

[5] B. Cross, C. Barraud, C. Picard, L. Léger, F. Restagno, and É. Charlaix, Wall slip of complex fluids: Interfacial friction versus slip length, Phys. Rev. Fluids 3, 062001 (2018).

[6] D. Camargo, J. A. de la Torre, R. Delgado-Buscalioni, F. Chejne, and P. Español, Boundary conditions derived from a microscopic theory of hydrodynamics near solids, J. Chem. Phys. 150, 144104 (2019).

[7] H. Nakano and S.-i. Sasa, Statistical mechanical expressions of slip length, J. Stat. Phys. 176, 312 (2019). 
[8] S. Goldstein, Note on the condition at the surface of contact of a fluid with a solid body, in Modern Developments in Fluid Dynamics (Dover, New York, 1965), pp. 676-680.

[9] G. K. Batchelor, An Introduction to Fluid Dynamics (Cambridge University Press, Cambridge, UK, 1967).

[10] J. S. Ellis and G. L. Hayward, Interfacial slip on a transverse-shear mode acoustic wave device, J. Appl. Phys. 94, 7856 (2003).

[11] G. M. Whitesides, The origins and the future of microfluidics, Nature (London) 442, 368 (2006).

[12] W. Sparreboom, A. van den Berg, and J. C. T. Eijkel, Principles and applications of nanofluidic transport, Nat. Nanotechnol. 4, 713 (2009).

[13] L. Bocquet and E. Charlaix, Nanofluidics, from bulk to interfaces, Chem. Soc. Rev. 39, 1073 (2010).

[14] A. Ajdari and L. Bocquet, Giant Amplification of Interfacially Driven Transport by Hydrodynamic Slip: Diffusio-Osmosis and Beyond, Phys. Rev. Lett. 96, 186102 (2006).

[15] Y. Ren and D. Stein, Slip-enhanced electrokinetic energy conversion in nanofluidic channels, Nanotechnology 19, 195707 (2008).

[16] D. J. Rankin and D. M. Huang, The effect of hydrodynamic slip on membrane-based salinity-gradientdriven energy harvesting, Langmuir 32, 3420 (2016).

[17] L. Fu, S. Merabia, and L. Joly, What Controls Thermo-osmosis? Molecular Simulations Show the Critical Role of Interfacial Hydrodynamics, Phys. Rev. Lett. 119, 214501 (2017).

[18] C. Neto, D. R. Evans, E. Bonaccurso, H.-J. Butt, and V. S. J. Craig, Boundary slip in Newtonian liquids: A review of experimental studies, Rep. Prog. Phys. 68, 2859 (2005).

[19] C. Cottin-Bizonne, B. Cross, A. Steinberger, and E. Charlaix, Boundary Slip on Smooth Hydrophobic Surfaces: Intrinsic Effects and Possible Artifacts, Phys. Rev. Lett. 94, 056102 (2005).

[20] A. Maali, T. Cohen-Bouhacina, and H. Kellay, Measurement of the slip length of water flow on graphite surface, Appl. Phys. Lett. 92, 053101 (2008).

[21] E. Secchi, S. Marbach, A. Niguès, D. Stein, A. Siria, and L. Bocquet, Massive radius-dependent flow slippage in carbon nanotubes, Nature (London) 537, 210 (2016).

[22] P. A. Thompson and S. M. Troian, A general boundary condition for liquid flow at solid surfaces, Nature (London) 389, 360 (1997).

[23] D. M. Huang, C. Sendner, D. Horinek, R. R. Netz, and L. Bocquet, Water Slippage versus Contact Angle: A Quasiuniversal Relationship, Phys. Rev. Lett. 101, 226101 (2008).

[24] V. P. Sokhan and N. Quirke, Slip coefficient in nanoscale pore flow, Phys. Rev. E 78, 015301(R) (2008).

[25] S. K. Kannam, B. D. Todd, J. S. Hansen, and P. J. Daivis, Slip length of water on graphene: Limitations of non-equilibrium molecular dynamics simulations, J. Chem. Phys. 136, 024705 (2012).

[26] L. Bocquet and J.-L. Barrat, On the Green-Kubo relationship for the liquid-solid friction coefficient, J. Chem. Phys. 139, 044704 (2013).

[27] K. Huang and I. Szlufarska, Green-Kubo relation for friction at liquid-solid interfaces, Phys. Rev. E 89, 032119 (2014).

[28] B. Ramos-Alvarado, S. Kumar, and G. P. Peterson, Hydrodynamic slip length as a surface property, Phys. Rev. E 93, 023101 (2016).

[29] S. Nakaoka, Y. Yamaguchi, T. Omori, and L. Joly, Molecular dynamics analysis of the friction between a water-methanol liquid mixture and a non-polar solid crystal surface, J. Chem. Phys. 146, 174702 (2017).

[30] S. Nakaoka, Y. Yamaguchi, T. Omori, and L. Joly, Extraction of the solid-liquid friction coefficient between a water-methanol liquid mixture and a non-polar solid crystal surface by Green-Kubo equations, Mech. Eng. Lett. 3, 17 (2017).

[31] A. Sam, R. Hartkamp, S. K. Kannam, and S. P. Sathian, Prediction of fluid slip in cylindrical nanopores using equilibrium molecular simulations, Nanotechnology 29, 485404 (2018).

[32] P. Simonnin, V. Marry, B. Noetinger, C. Nieto-Draghi, and B. Rotenberg, Mineral- and ion-specific effects at clay-water interfaces: Structure, diffusion, and hydrodynamics, J. Phys. Chem. C 122, 18484 (2018).

[33] H. Nakano and S.-i. Sasa, Microscopic determination of macroscopic boundary conditions in Newtonian liquids, Phys. Rev. E 99, 013106 (2019).

[34] C. Herrero, T. Omori, Y. Yamaguchi, and L. Joly, Shear force measurement of the hydrodynamic wall position in molecular dynamics, J. Chem. Phys. 151, 041103 (2019). 
[35] The bulk viscoelastic behavior of a liquid has been long recognized since Maxwell [53]. For complete reference, see [54-56].

[36] G. McHale, R. Lücklum, M. I. Newton, and J. A. Cowen, Influence of viscoelasticity and interfacial slip on acoustic wave sensors, J. Appl. Phys. 88, 7304 (2000).

[37] X. Qiao, X. Zhang, Y. Tian, and Y. Meng, Modeling the response of a quartz crystal microbalance under nanoscale confinement and slip boundary conditions, Phys. Chem. Chem. Phys. 17, 7224 (2015).

[38] X. Qiao, X. Zhang, Y. Tian, and Y. Meng, Progresses on the theory and application of quartz crystal microbalance, Appl. Phys. Rev. 3, 031106 (2016).

[39] N. V. Priezjev, Molecular dynamics simulations of oscillatory Couette flows with slip boundary conditions, Microfluid. Nanofluid. 14, 225 (2013).

[40] K. Ogawa, H. Oga, H. Kusudo, Y. Yamaguchi, T. Omori, S. Merabia, and L. Joly, Large effect of lateral box size in molecular dynamics simulations of liquid-solid friction, Phys. Rev. E 100, 023101 (2019).

[41] H. J. C. Berendsen, A Student's Guide to Data and Error Analysis (Cambridge University Press, Cambridge, UK, 2011).

[42] A. R. Dexter and A. J. Matheson, Elastic moduli and stress relaxation times in liquid argon, J. Chem. Phys. 54, 203 (1971).

[43] T. Yamaguchi, Stress-structure coupling and nonlinear rheology of Lennard-Jones liquid, J. Chem. Phys. 148, 234507 (2018).

[44] P.-A. Mante, C.-C. Chen, Y.-C. Wen, H.-Y. Chen, S.-C. Yang, Y.-R. Huang, I. -Ju Chen, Y.-W. Chen, V. Gusev, M.-J. Chen, J.-L. Kuo, J.-K. Sheu, and C.-K. Sun, Probing hydrophilic interface of solid/liquidwater by nanoultrasonics, Sci. Rep. 4, 6249 (2014).

[45] P. Español and I. Zúñiga, Force autocorrelation function in Brownian motion theory, J. Chem. Phys. 98, 574 (1993).

[46] J. S. Hansen, B. D. Todd, and P. J. Daivis, Prediction of fluid velocity slip at solid surfaces, Phys. Rev. E 84, 016313 (2011).

[47] L. Joly, G. Tocci, S. Merabia, and A. Michaelides, Strong coupling between nanofluidic transport and interfacial chemistry: How defect reactivity controls liquid-solid friction through hydrogen bonding, J. Phys. Chem. Lett. 7, 1381 (2016).

[48] H. Oga, Y. Yamaguchi, T. Omori, S. Merabia, and L. Joly, Green-Kubo measurement of liquid-solid friction in finite-size systems, J. Chem. Phys. 151, 054502 (2019).

[49] H. Hoang and G. Galliero, Local viscosity of a fluid confined in a narrow pore, Phys. Rev. E 86, 021202 (2012).

[50] M. Morciano, M. Fasano, A. Nold, C. Braga, P. Yatsyshin, D. N. Sibley, B. D. Goddard, E. Chiavazzo, P. Asinari, and S. Kalliadasis, Nonequilibrium molecular dynamics simulations of nanoconfined fluids at solid-liquid interfaces, J. Chem. Phys. 146, 244507 (2017).

[51] By this forcing method, the wall shear stress in the MD simulation results should match the continuum predictions but the uniform pressure gradient assumed in Eq. (10) is not guaranteed near the walls. Under the simulation conditions here, however, the discrepancy seems negligible considering that the MD simulation results fall onto parabolic curves well.

[52] A. Alizadeh Pahlavan and J. B. Freund, Effect of solid properties on slip at a fluid-solid interface, Phys. Rev. E 83, 021602 (2011).

[53] J. C. Maxwell, IV. On the dynamical theory of gases, Philos. Trans. R. Soc. London 157, 49 (1867).

[54] J. P. Boon and S. Yip, Molecular Hydrodynamics (Dover, New York, 1991).

[55] D. J. Evans and G. Morriss, Statistical Mechanics of Nonequilibrium Liquids, 2nd ed. (Cambridge University Press, Cambridge, UK, 2008).

[56] J.-P. Hansen and I. R. McDonald, Theory of Simple Liquids, 4th ed. (Elsevier, Amsterdam, 2013). 